\title{
The Annual Meeting of the German Society of Neuroradiology
}

\author{
M. Knauth
}

Published online: 12 May 2012

(C) The Author(s) 2012. This article is published with open access at Springerlink.com

Dear Colleagues and friends of neuroradiology,

The annual meeting of the German Society of Neuroradiology, neuroRAD, has become the largest European meeting in the field of neuroradiology. The last three annual meetings have achieved this by offering an (apparently convincing) assortment of teaching and scientific news.

This year's main "interventional" topics include intra-cranial aneurysms, intra-arterial stroke therapy and endovascular treatment of stenosis of the intra- and extracranial arteries supplying the brain. Furthermore, the popular session "What's new in neuroradiology?" will get its own "interventional" slot, thus providing a comprehensive update on diagnostic and therapeutic neuroradiology.

Precise knowledge of neuroanatomy is a prerequisite for accurate diagnosis in neuroradiology. Therefore, one of the field's main focuses of teaching is the neuroanatomy of cortical, deep brain and brain stem structures, offering numerous tips and tricks for the identification of crucial anatomical landmarks.

A further topic is our aging society, where the focus lies on neurodegeneration, dementia and stroke.
Brain tumors and the "new" diagnostic tools available here, as well as a session on "pitfalls" in brain tumor diagnosis, round up this part of the programme.

One of the mainstays of the congress is a dedicated program for radiology technicians, without whom we would be lost and whose skills co-determine the quality of our own work.

Abstract submission is open: further information can be obtained by visiting www.neurorad.de.

The German Society of Neuroradiology would like to welcome you as a participant at its upcoming 47th meeting from the 11th to the 13th of October 2012 in Cologne.

Olav Jansen

President of the German Society of Neuroradiology

Michael Knauth

Congress President

Open Access This article is distributed under the terms of the Creative Commons Attribution License which permits any use, distribution, and reproduction in any medium, provided the original author(s) and the source are credited. 